\title{
Analysis on the Application of Cultural Orientation Furnishings in Contemporary Themed Dining Space Design
}

\author{
Xin $\mathrm{Fu}^{1, *}$ Zhixiang $\mathrm{Jin}^{1}$ \\ ${ }^{1}$ College of Art and Design, Wuhan Textile University, Wuhan, Hubei, China \\ "Corresponding author. Email: $1073419084 @$ qq.com
}

\begin{abstract}
The rapid development of China's social economy has brought about the continued prosperity of the catering industry. The thematic catering industry that has emerged in recent years has further expanded its multi-dimensional culture. People are paying more and more attention to the cultural expression of the indoor design of dining spaces, and as an indispensable part, it has also been actively researched and used by the catering industry. Combined with actual design cases in China and foreign countries, this paper relates to the cultural development background and trend of contemporary themed dining spaces, analyzes the meaning of furnishings in contemporary themed dining spaces and the expression of furnishings with cultural orientation in indoor spaces, and explains its application scope and value in contemporary themed dining space.
\end{abstract}

Keywords: indoor design, themed dining space, furnishings, cultural orientation

\section{INTRODUCTION}

With the development and progress of Chinese society, today's modern production and lifestyles are quietly changing people's living habits. The continuous emergence of new technologies, new crafts and new ideas makes people's requirements for the dining environment and dining quality increasingly higher. People are no longer confined to a single dining need, but are pursuing a variety of demand factors such as rich negotiation needs, social needs, psychological needs, and emotional needs. People's pursuit of more diverse and richer dining experience has led to the emergence of themed dining spaces and made them gradually favored by people.

\section{MULTI-DIMENSIONAL CULTURAL ORIENTATION IN CONTEMPORARY THEMED DINING SPACES}

Nowadays, the understanding of furnishings in the furnishing design of many restaurants in China is still only in the decorative meaning of the dining space, and very similar furnishings can also be found in some dining space design works. Obviously, these dining spaces lack accurate thinking about the restaurant concept, and fail to reflect the uniqueness and artistry of their cultural connotations. Undoubtedly, the furnishings in contemporary themed restaurants are no longer purely decorative. They are more about combining cultural and artistic connotations to improve the quality of the overall dining environment and bring people a richer spiritual experience.

The furnishings in the field are used to beautify or strengthen the visual effect of the environment, and have ornamental value or cultural significance. Among them, the cultural orientation furnishings have the characteristics of clear cultural symbols, expressing certain cultural heritage and ideological connotation. In contemporary themed dining spaces, for the viewers, cultural orientation furnishings are not only important symbols to experience the theme culture of the dining space, but also fill the gaps in the viewer's spiritual and cultural needs outside of dining and satisfy the dual demands of material and spirit; for merchants, the cultural orientation in themed catering spaces has become an important symbol that embodies brand concepts and enhances the value of their catering brands. It can be seen that the cultural orientation in contemporary themed dining spaces is of vital importance to consumers or merchants. 


\section{THE RELEVANCE OF THE CULTURAL ORIENTATION OF FURNISHINGS IN CONTEMPORARY THEMED DINING INDOOR DESIGN}

\section{A. Cultural connotation and symbolic meaning of furnishings in furnishing design}

The concept of the themed restaurant is to enjoy the food while also experiencing its intended theme culture, and the cultural orientation furnishings in the furnishing design play a role in creating the indoor style of the themed restaurant and highlighting the theme culture of the restaurant. The furnishings in the indoor design are an important part of it, as well as an important element to enrich and optimize the space experience. Different architectural spaces have different indoor designs, and different design concepts match different furnishings. The cultural and artistic features contained in the furnishings have re-directed to its indoor design concepts, highlighting the entire indoor space, and making the space contain rich emotional colors and unique artistic beauty. The cultural understanding and expression of furnishings comes from the characteristics of cultural diversity and tolerance. The differences between cultures allow furnishings to have different charms, drawing design inspiration from cultural collisions, evolving into a unique and culturally significant indoor style, and making furnishings unique and attractive under the influence of diverse cultures.

As one of the important parts of indoor design, the presence of furnishings in the corresponding space is a cultural manifestation, which embodies the designer's design philosophy and cultural intentions, and conveys its own beauty. The materials, colors, structures, and patterns of the furnishings all convey cultural emotions from different angles, allowing people to discover the cultural atmosphere contained in the space and generate emotional resonance. This resonance connects objects and people, space and spirit, and conveys the charm of culture and art. Multi-sensory stimuli such as vision, hearing, smell, etc. directly affect people's intuitive experience of the space environment, and are also the direct interaction between people and space furnishings. The cultural information contained in the furnishings is diverse, and the artistic value of the furnishings is also unique. The cultural aesthetic meaning it conveys will also make viewers have different experiences and feelings, and new meaning will be produced in the process of information transmission. Just like appreciating the same work of art, some people think that it expresses a feeling of "simplicity", while some people think that such a performance is meaningless. Facing furnishings and artworks, people's subjective feelings are diverse and unique. This indeterminate perception of difference requires that the furnishings have a unified cultural concept and cultural background in the design. Only in this way, the information that the viewer feels in the space will be clearer and more consistent, instead of being vague and divergent.

From the perspective of cultural aesthetics, the symbolic meaning of furnishings is an important way of artistic expression. The significance of the furnishings in the dining space is not only to reflect its own artistic appreciation value, but also to interact with the space and the environment through several furnishings, interact and jointly affect the cultural and artistic atmosphere of the entire indoor space. At the same time, they deliver unique and more directional cultural and artistic feelings to the viewers. Of course, furnishings in themed dining space are also different from furnishings in art galleries and museums whose main purpose is to show and display. Although the furnishings in the two spaces have artistic value and cultural attributes, the emphasis between the two is not exactly the same. The furnishings in art galleries, museums and other cultural exhibition halls pay more attention to show their own cultural and artistic value, and are not closely related to the space in which they are located. In other words, the space is "serving" the furnishings to a large extent, and it won't consider too much about whether the furnishings are reasonable in the space. The meaning of the furnishings in the themed dining space is completely opposite. It is more about coordinating the integrity of the space, and emphasizing the unity and rationality of the entire indoor space culture, that is, furnishings "serve" the space. From a semiotic point of view, there is a certain connection between the furnishings and the dining space. The symbolic meaning of the furnishings does not exist in isolation, but the series combination of several symbolic meanings of different furnishings in the space together constitute the cultural value of the space. The value of furnishings is not only to bring sensory stimulation to the viewers, but also to establish cultural connections with the audience, and to build a variety of sensory and spiritual experiences in the themed dining space.

\section{B. Cultural expression of contemporary themed dining space in China and foreign countries}

China has been a country with a diverse food culture since ancient times. Nowadays, there is no shortage of themed restaurants that integrate traditional Chinese cultural meanings into the dining space to create a dining atmosphere with traditional Chinese regional characteristics, allowing viewers to have a new dining experience and feelings. The new Beijing cuisine themed restaurant "Dianmao Small Courtyard" in Beijing is a themed restaurant with the charm of the old Beijing Hutong quadrangle dwellings with blue bricks and gray tiles as well as green windows and vermilion doors. As shown in "Fig. 1", the space retains the spatial characteristics of the old Beijing quadrangle 
dwellings, and the color continues the poetry of blue bricks and gray tiles, and flourishing flowers and withering leaves. The whole space is imaginary and real properly, and the form is simplified. The modern, concise and clear design concept incorporates traditional element characteristics to outline the unique scenery in the indoor space. For the Chinese style charm of the small courtyard, from the main entrance of the restaurant to the internal space, the color of the entire space gradually decreases from thick to light. The entrance is the most welcoming, festive, and also the most preferred vermilion color of the Chinese. From the wall to the top, it forms a house-like entrance reception area, which is also like a yard screen wall.



Fig. 1. "Dianmao Small Courtyard" restaurant entrance.

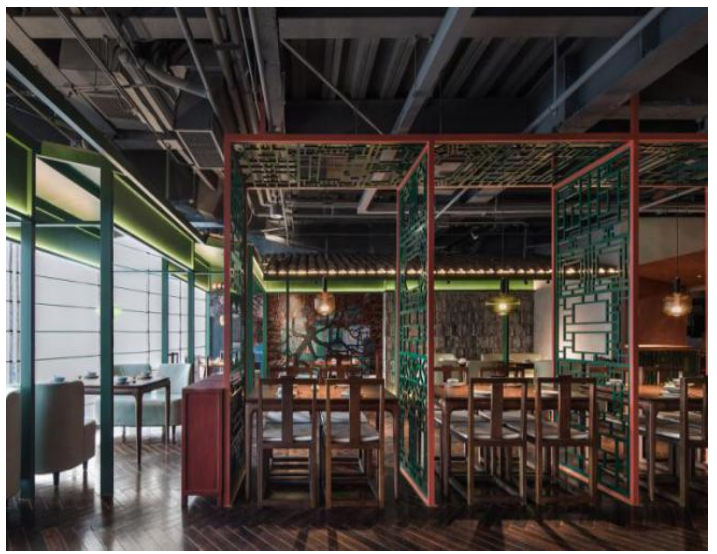

Fig. 2. Dining area of "Dianmao Small Courtyard" restaurant.

Inside the small courtyard, it has the blue bricks, gray tiles, green windows, and vermilion doors, and in terms of its form, it has the shadow of quadrangle dwellings, but it is not exactly the same. The unique cultural symbols of the small courtyard become the continuation of the past and the modern through deconstruction of form, color refinement, and modern simple design language. The corridors and houses erected by the columns of the "disappeared" courtyards are replaced by modern materials. The green metal spray-painted square tube frames a herringbone roof, strewing at random with gray tiles being laid. It resembles courtyards and corridors, which is arranged symmetrically with the surroundings, showing the beauty of etiquette. The window panes with unique Chinese patterns not only distinguish the space, but also reveal the cultural charm of the Chinese style, which balances the function and the artistic form. As shown in "Fig. 2", the four groups of dining areas separated by green metal panes in the middle of the courtyard are relatively independent and interactive. The inside and outside, obstruction and separation, and emptiness and reality are both a scene in the courtyard and a part of its function.

Various modern art trends all originated abroad, and different art trends and design styles have been produced under the influence of different art movements and artistic trends. The design research of foreign themed dining spaces and the application research of furnishings in indoor spaces are earlier than those in China. The cultural expression of furnishings in themed dining spaces is influenced by the western culture and is different from Chinese cultural expressions.

A Tuna Boutique Restaurant located in Barcelona, Spain, is a tuna-themed boutique restaurant that aims to showcase a gourmet space dedicated to enjoying red tuna through design, and promote the high-quality red tuna food material, while also letting the public know about its brand. The entire indoor space of the restaurant is inspired by tuna's bodily form, texture, color and flesh quality, creating a feeling of space like being in the deep sea. As shown in "Fig. 3", the dark blue indoor space with abstract "fish school" art furnishings makes people feel that as if you were in the depths of the seabed, with the graceful changes in the surrounding lights, making the feeling of immersion in the ocean stronger. The translucent curtains in front of the glass curtain wall increase the deep intangible sense of the indoor space, and the light texture also softens the space experience in the visual sense. The restaurant's seats are ingeniously chosen with red inner face seats similar to tuna meat, which form a strong contrast with the restaurant's dark blue hue. As shown in "Fig. 4", the tile veneering used in the restaurant is clean and easy to take care of. At the same time, referring to the luminous characteristics of tuna blue and silver scales, the tiles can just satisfy this trait. To this end, the restaurant designs tile veneering in different tones and shapes for the floor and walls. The effect is low-key but unique, showing a mysterious and dreamy feeling. 


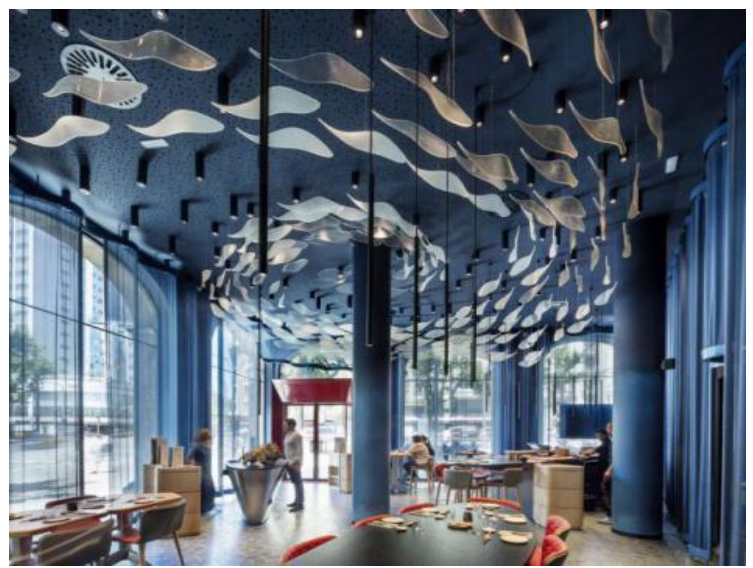

Fig. 3. The indoor Dining Area 1 of Tuna Boutique Restaurant.

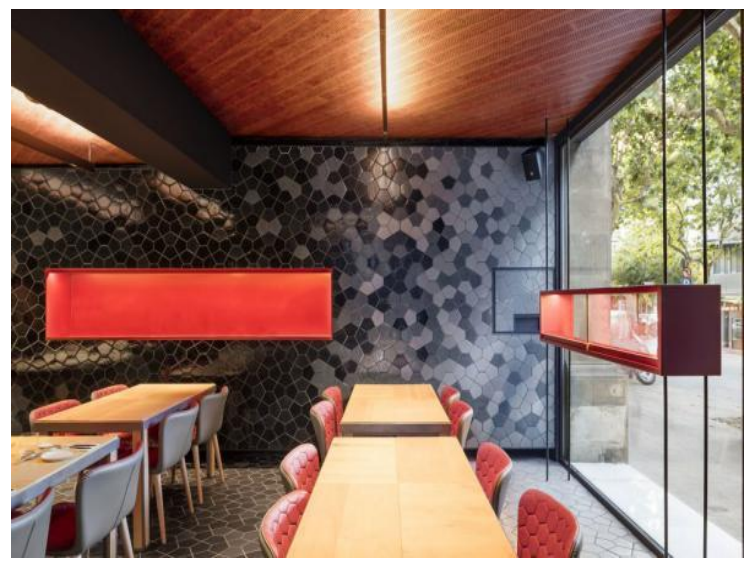

Fig. 4. The indoor Dining Area 2 of Tuna Boutique Restaurant.

\section{Expression of cultural orientation furnishings in indoor design}

The role of cultural orientation furnishings in indoor space is mainly reflected in the following three aspects: one is to enhance the spatial cultural form aesthetic feeling. Form aesthetic feeling is an important factor in judging the quality of indoor space design, and it is also the focus of the overall indoor environment. While in the themed dining space, the importance of culture is placed in an important position, and the furnishings that reflect cultural features become the center of vision. Factors such as the cultural aesthetic attributes of the furnishings and the impact of the visual form greatly enhance the overall cultural atmosphere of the themed dining space, enhance the cultural characteristics of the space, and improve the taste of the restaurant. Compared with ordinary dining spaces, themed dining spaces have more uniform style requirements and more diverse spaces. The rational use of furnishings not only echoes the theme style in visual perception, but also enhances the overall cultural tone of the space in terms of cultural form. Similarly, furnishings can also play a practical and functional role, such as signs and instructions, which not only play a practical guiding role, but also do not destroy the overall cultural and artistic beauty of the space.

The second is to enhance the sense of substitution of theme culture. Any culture and art are diverse and tolerant. The difference in people's aesthetics makes the classic and modern, and traditional and fashionable go hand in hand. It is impossible to jump to a conclusion about who is more influential and superior. The modern and fashionable do not mean creating novelty, and the classic and traditional do not mean obsolescence. The themed dining space does not appear to meet all the people with different aesthetic needs, but to grasp the target group in a targeted manner. Audiences with specific aesthetic needs need to experience an artistic atmosphere with a strong and clear cultural connotation in the indoor space. At this time, the role of building cultural atmosphere played by the indoor stuff is quite limited and not rich. Therefore, the furnishings play a vital role. Reasonably arranged furnishings are dotted in the space, conveying cultural information on the premise of satisfying its basic functions, and enhancing the audience's emotional experience in the space, that is, enhancing the sense of cultural substitution in the themed dining space.

The third is to enrich the spatial hierarchy. With the continuous development of modern science and technology, new technologies and new materials continue to appear. Modern building materials are no longer limited to traditional materials such as cement, steel, glass, and wood. The application of composite materials is also increasing. This not only brings new changes to the architectural design, but also brings unlimited possibilities to the indoor space. Sturdy, durable, malleable, and colorful materials build a variety of spaces. Different indoor spaces give people different feelings: the traditional indoor space tends to be tough but lacks change. In the treatment of space, natural materials can be used to soften the space, and natural plants or fabrics can also be used to improve the space, and the space then can be softened visually. The soft and hard materials complement each other and enrich the spatial hierarchy. The trendy indoor space is rich in changes but lacks stability. Also in the space processing, furnishings can be used to set the visual center, stabilize the visual center of gravity, and coordinate the overall feeling of the space.

\section{THE USE OF CULTURAL ORIENTATION FURNISHINGS IN CONTEMPORARY THEMED DINING SPACES}

The cultural orientation furnishings play the role of "the finishing touch" in contemporary themed dining spaces. Unlike ordinary decorations, cultural orientation furnishings can be artworks or installations that do not 
have specific use functions, such as decorative paintings, art installations, etc.; they can also be furnishings with actual use functions, such as tables, chairs, artistic lamps, etc. The role of cultural orientation furnishings is to highlight the theme atmosphere of the restaurant. People in the space experience the cultural concepts brought by the restaurant, and experience the charm of culture and art while taking meals.

\section{A. The decorative application of cultural orientation furnishings}

The cultural orientation furnishings in the dining space achieve the purpose of expanding the cultural interest of the space, creating a visual center in the regional space, and enhancing the sense of form of the space model through its decorative features. As shown in "Fig. 5", the restaurant with the theme of "tuna" focuses the visual center of the indoor space on the abstract "school of fish". In the deep blue background like the ocean, with the appropriate light source, it creates a strong "deep sea" experience. The abstract "schools of fish" floating in the air give the viewers a flexible and lightsome visual experience. However, as an art installation of the restaurant, it does not have actual use function, but it plays a key role in the creation of the theme atmosphere of the space and the cultural interest of the restaurant. As shown in "Fig. 6", in the restaurant "Dianmao Small Courtyard", the blue tiles and gray bricks of the outdoor roof of the courtyard are introduced into the indoor to form symbolic decorative elements. The geometric shape of the door opening at the entrance is extracted from the geometric features of the facade of the courtyard, combined with vermilion and white colors, presenting a modern and concise style. The traditional blue tile and gray brick sloping roof is matched with the modern geometric door opening to form a blend of traditional and modern styles. At the same time, it enhances the sense of form of the modeling style in the indoor space, so that the space has a different kind of vitality and beauty, and achieves a balance between tradition and modernity.

\section{B. Functional use of cultural orientation furnishings}

Whether it is decorations with no specific use function or characteristic furniture with cultural symbols with use function in the dining indoor space, it is the carrier of the style language of the entire dining indoor space. Among them, the furnishings with cultural symbols bears people's needs for space use in function; in terms of the culture of furnishings, the corresponding cultural information is transmitted through symbol elements such as furniture shape, material, color, etc., so that it is integrated with the themed restaurant design concept in terms of cultural implication. It is not so much the characteristic furniture of the restaurant, as it is a furnishing in the restaurant that expresses cultural orientation. As shown in "Fig. 7", the themed restaurant of "Dianmao Small Courtyard" of special Beijing flavour extracts the symbolic element structure of old Beijing quadrangle dwellings, and uses it in the restaurant space. The decorative patterns of the window grille and the reception table wall in the picture all adopt the pattern characteristics of quadrangle dwellings' panes, pointing the cultural intention to the life mood of old Beijing quadrangle dwellings, and highlighting the emotional atmosphere of the entire restaurant space. The color combination of red and green is just right, which makes people easily think of the matching hue of the green windows and vermilion doors of the quadrangle dwellings. Functionally, the setting of the window grille organically divides the originally empty large space into several small dining spaces. The hollow grille not only makes the divided space appear transparent and not depressing, but also can effectively ensure the privacy of people when dining. Combined with the cultural expression of the grille patterns and the reasonable collocation of colors, it is organically integrated into the entire style, avoiding the discomfort caused by the sudden division of the space.

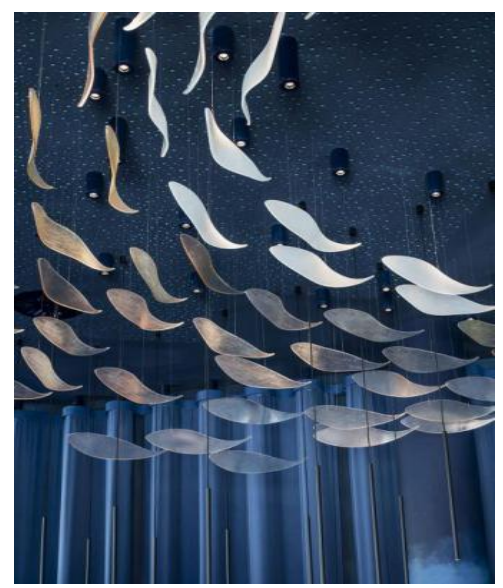

Fig. 5. Tuna Boutique Restaurant art installation

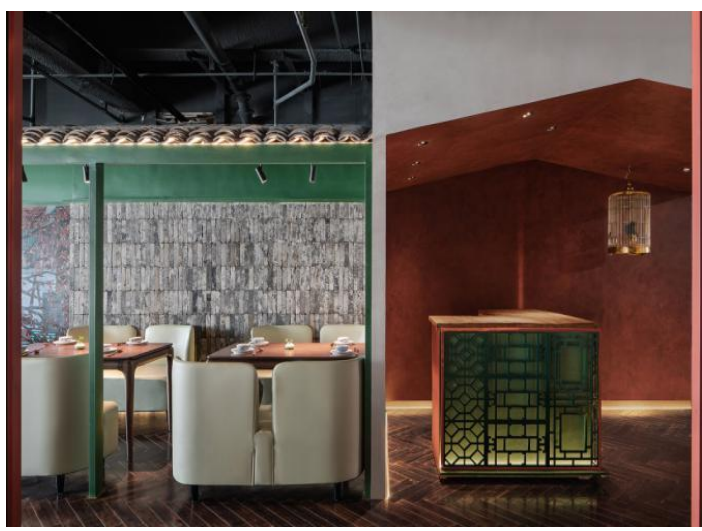

Fig. 6. "Dianmao Small Courtyard" restaurant space geometry. 


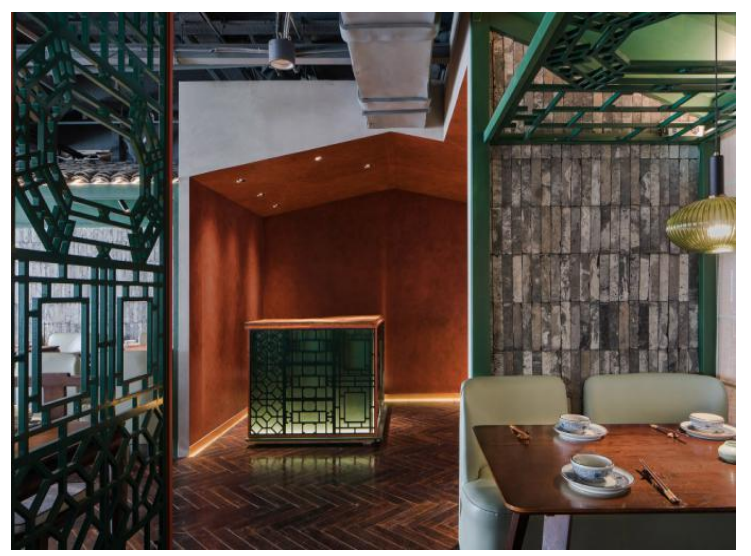

Fig. 7. "Dianmao Small Courtyard" restaurant furnishing details.

\section{CONCluSion}

The use of cultural orientation furnishings in dining spaces is becoming more and more important, and the fundamental reason lies in contemporary people's spiritual pursuit of a higher level of culture against the background of rapid social and economic development, and it also conforms to the trend of contemporary style design. Cultural orientation furnishings cover a wide range and are one of the most important components for contemporary themed dining spaces. This paper analyzes and studies the actual cases in China and foreign countries, and analyzes the cultural expression in contemporary themed dining spaces and the application of cultural orientation furnishings in contemporary themed dining spaces. The cultural orientation furnishings highlight the cultural atmosphere of the overall space, and the theme style symbols are more obvious, which are more in line with the spiritual and cultural needs of viewers. Therefore, the expression of cultural orientation furnishings in contemporary themed dining spaces is extensive and important. In practical applications, the understanding of furnishings in themed dining spaces should be more focused on the shaping and expression of cultural and spiritual levels.

\section{References}

[1] Cao Xiangzhe. Indoor furnishing design [M]. Beijing: Posts and Telecom Press. 2015.8 (in Chinese)

[2] Liu Man. Dining and cultural space design [M]. Chongqing: Southwest Normal University Press. 2004 (in Chinese)

[3] Meng Yilun. Research on the theme culture application of furnishing design in dining spaces [D]. Jilin University. 2018 (in Chinese)

[4] Li Hongfeng. Application research of furnishings in dining environment design [D]. Anhui Architecture University. 2018 (in Chinese)

[5] Wang An. The creation of imagery - an analysis of the thematic design of indoor furnishings [J]. Urban Architecture. 2019 (in Chinese)

[6] Yu Zhaojiang. Application of indoor furnishing art [J]. Science $\&$ Technology Economic Guide. 2019 (in Chinese) 